\title{
Pattern formation in driven colloidal mixtures: tilted driving forces and re-entrant crystal freezing
}

\author{
J Dzubiella and H Löwen \\ Institut für Theoretische Physik II, Heinrich-Heine-Universität Düsseldorf, Universitätsstraße 1, \\ D-40225 Düsseldorf, Germany \\ E-mail: joachim@thphy.uni-duesseldorf.de
}

Received 30 January 2002, in final form 17 April 2002

Published 27 September 2002

Online at stacks.iop.org/JPhysCM/14/9383

\begin{abstract}
The influence of an external field acting differently on the two constituents of a binary colloidal mixture performing Brownian dynamics is investigated by computer simulations and a simple theory. In our model, half of the particles (A particles) are pulled by an external force $\vec{F}^{(A)}$ while the other half (B particles) are pulled by an external force $\vec{F}^{(B)}$. If $\vec{F}^{(A)}$ and $\vec{F}^{(B)}$ are parallel and the fieldfree state is a mixed fluid, previous simulations (Dzubiella J et al 2002 Phys. Rev. E 65 021402) have shown a nonequilibrium pattern formation involving lanes of A or B particles only, which are sliding against each other in the direction of the external forces. In this paper, we generalize the situation both to nonparallel external forces and to field-free crystalline states. For nonparallel forces, lane formation is also observed but with an orientation tilted with respect to the external forces. If the field-free state is crystalline, a continuous increase of the parallel external forces yields a novel re-entrant freezing behaviour: the crystal first melts mechanically via the external force and then recrystallizes into demixed crystalline lanes sliding against each other.
\end{abstract}

\section{Introduction}

When brought into nonequilibrium, physical systems may spontaneously exhibit many different kinds of pattern formation (for recent reviews see $[1,2]$ ) which are much richer than the traditional phase transitions in equilibrium systems. While the latter are by now well understood in terms of microscopic theories and simulations [3-6], full microscopic theories operating on a particle-resolved level for nonequilibrium situations still represent a major challenge. In this paper we study a 'microscopic' system designed to model binary colloidal suspensions in an external field which is an off-lattice version [7] of a diffusive system in an external driving field. Colloidal dispersions indeed represent excellent model systems, which can be brought into nonequilibrium [8,9] via external fields in a controlled way [10] and can be studied experimentally with a real-space resolution of an interparticle distance [11]. 
Another complementary realization of a driven diffusive off-lattice system is pedestrian motion in pedestrian zones [12-14].

In our model, we consider an equimolar binary mixture of so-called A and B particles. The particles are identical as far as their mutual interaction is concerned, which we model via a Yukawa pair potential having charged colloidal dispersions in mind. The A and B particles, however, respond differently to the external field applied: A particles feel an external force $\vec{F}^{(A)}$ while B particles are driven by a different force $\vec{F}^{(B)}$. Completely overdamped Brownian dynamics (with hydrodynamic interactions neglected) are assumed $[15,16]$ for the colloidal motion. The case of parallel forces where $\vec{F}^{(A)}=-\vec{F}^{(B)}$ was investigated recently by computer simulation by us and Hoffmann [17] via nonequilibrium Brownian dynamics computer simulations in the case where the field-free thermodynamic equilibrium state was a mixed fluid. As a result, above a critical strength of the external force, the system exhibits a transition towards lane formation. The lanes comprise bundles of particles of the same kind (A or B) and are parallel to the driving field. This nonequilibrium phase separation [18] results from a slipstream effect caused or transported by the interparticle interactions. The critical force can be theoretically estimated by setting the external force to a typical interaction force resulting from a pairwise potential $V(r)$ between the particles. Experimental evidence for such an instability has been accumulated in sedimentation dynamics of bidisperse suspensions [19-22].

In this paper, we generalize the set-up previously studied in [17] in the following two directions. First, we study the case where the two external forces $\vec{F}^{(A)}$ and $\vec{F}^{(B)}$ are not parallel. Second, we study - for parallel forces - the case where the equilibrium field-free state is crystalline. For nonparallel forces, lane formation also occurs. The direction of the lanes, however, is tilted with respect to the driving fields. In more detail, lanes are directed along the difference vector $\vec{F}^{(B)}-\vec{F}^{(A)}$ of the two external forces. For a randomly occupied crystal, on the other hand, a two-stage transition shows up: first, above a threshold, the external field melts the solid mechanically. Upon increasing the external field strength further, a reentrant freezing transition is discovered. The resulting crystalline structure involves completely demixed A and B solids sliding against each other, similar to the fluid lane formation.

This paper is organized as follows. In section 2, we define the model used and describe briefly our simulation technique. Results for nonparallel external forces are presented in section 3. The solid-fluid-solid re-entrant behaviour is discussed in section 4. Conclusion are given in section 5. In particular, we discuss a possible verification of our predictions in experiments.

\section{The model}

In our model [17], we consider a symmetric binary colloidal mixture comprising $2 N$ Brownian colloidal particles in $d=2$ spatial dimensions. Half of them are particles of type $\mathrm{A}$ and the other half is of type $\mathrm{B}$, with partial number densities $\rho_{A}=\rho_{B}=\rho / 2$. The colloidal suspension is held at fixed temperature $T$ via the bath of microscopic solvent particles. Two colloidal particles interact via an effective Yukawa pair potential

$$
V(r)=V_{0} \sigma \exp [-\kappa(r-\sigma) / \sigma] / r,
$$

where $r$ is the centre-to-centre separation, $V_{0}$ is an energy scale and $\sigma$ is the particle diameter as a length scale. This is a valid model for charge-stabilized suspensions in three [23] and two [24-26] spatial dimensions.

The dynamics of the colloids is overdamped Brownian motion. The friction constant $\xi=3 \pi \eta \sigma$ (with $\eta$ denoting the shear viscosity of the solvent) is assumed to be the same for both $\mathrm{A}$ and $\mathrm{B}$ particles. The constant external force acting on the $i$ th particle, $\vec{F}_{i}$, is different 
for the two constituents of the binary mixture. It is $\vec{F}_{i}=\vec{F}^{(A)}$ for A particles and $\vec{F}_{i}=\vec{F}^{(B)}$ for B particles.

The stochastic Langevin equations for the colloidal trajectories $\vec{r}_{i}(t)(i=1, \ldots, 2 N)$ read as

$$
\xi \frac{\mathrm{d} \vec{r}_{i}}{\mathrm{~d} t}=-\vec{\nabla}_{\vec{r}_{i}} \sum_{j \neq i} V\left(\left|\vec{r}_{i}-\vec{r}_{j}\right|\right)+\vec{F}_{i}+\vec{F}_{i}^{(\mathrm{R})}(t) .
$$

The right-hand side includes all forces acting on the colloidal particles, namely the force resulting from inter-particle interactions, the external constant force and the random forces $\vec{F}_{i}^{(\mathrm{R})}$ describing the collisions of the solvent molecules with the $i$ th colloidal particle. The latter are Gaussian random numbers with zero mean, $\overrightarrow{\vec{F}}_{i}^{(\mathrm{R})}=0$, and variance

$$
\overline{\left(\vec{F}_{i}^{(\mathrm{R})}\right)_{\alpha}(t)\left(\vec{F}_{j}^{(\mathrm{R})}\right)_{\beta}\left(t^{\prime}\right)}=2 k_{B} T \xi \delta_{\alpha \beta} \delta_{i j} \delta\left(t-t^{\prime}\right) .
$$

The subscripts $\alpha$ and $\beta$ represent the two Cartesian components and $k_{B} T$ is the thermal energy. In the absence of an external field, the model reduces to a two-dimensional Brownian Yukawa fluid in equilibrium, which has been extensively investigated as far as structural and dynamical equilibrium correlations and freezing transitions are concerned [25-27].

We solve the Langevin equations of motion by Brownian dynamics simulations $[15,28,29]$ using a finite time-step and the technique of Ermak $[3,30]$. We observed the system running into a steady-state after a transient relaxation. We put $N=250 \mathrm{~A}$ and $250 \mathrm{~B}$ particles into a square cell of length $\ell$ with periodic boundary conditions. The total colloidal number density is $\rho=2 N / \ell^{2}$. The typical size of the time-step was $0.003 \tau_{B}$, where $\tau_{B}=\xi \sigma^{2} / V_{0}$ is a suitable Brownian timescale. We simulated typically $2 \times 10^{4}$ time-steps, which corresponds to a simulation time of $60 \tau_{B}$. After an initial relaxation period of $20 \tau_{B}$, statistics was gathered in the steady state.

\section{Nonparallel external forces}

\subsection{General argument for tilted lane formation}

Let us first recapitulate what is known for parallel external forces $\vec{F}^{(A)}$ and $\vec{F}^{(B)}$ : in [17], it was shown that lane formation occurs involving either A or B particles which are sliding against each other in the field direction. In the lane involving A particles only all these particles are drifting with a global velocity $\vec{F}^{(A)} / \xi$, while opposite regions which involve B particles are streaming with the overall velocity $\vec{F}^{(B)} / \xi$. By subtracting the overall velocity using a Galilei transformation one readily sees that within the completely separated lanes, equilibrium Boltzmann statistics is realized. The system just separates into two different equilibrium states which are drifting relative to each other. Physically, the formation of lanes is generated by collisions of A against B particles pushed by the external force, which dynamically separates A and B particles until completely demixed lanes are formed. A similar lane formation for sheared granular matter was found via molecular dynamics simulation in [31]. The formation of lanes is a sharp first-order nonequilibrium phase transition occurring if the external field difference $\left|\vec{F}^{(A)}-\vec{F}^{(B)}\right|$ exceeds a critical value. A Galilei transformation also proves that only the relative velocity of A and B regions is relevant. Hence, without loss of generality, it is sufficient to study the special case $\vec{F}^{(A)}=-\vec{F}^{(B)}$.

For nonparallel external forces, the collisions between A and B particles are no longer central and the phase-separated structure will be different, in general. In order to obtain insight into the location of an interface between two completely demixed regions involving A and B particles only, we first make a simple continuum argument: consider a (one-dimensional) 


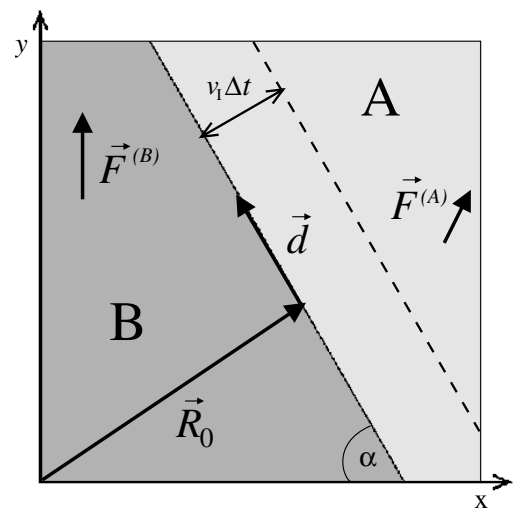

Figure 1. One-dimensional interface separating a region containing A particles (light grey) and B particles only (dark grey). The direction of the interface is $\vec{d} . \vec{R}_{0}$ points to the interface. The new interface after a time $\Delta t$ at distance $v_{\mathrm{I}} \Delta t$ from the original one is shown as a dashed line.

interface between an $\mathrm{A}$ and a $\mathrm{B}$ region with a direction described by a two-dimensional vector $\vec{d}$ (see figure 1). The full interface position can be parametrized by a set of vectors

$$
\vec{r}(s)=\vec{R}_{0}+s \vec{d}
$$

where $s$ is a real parameter and $\vec{R}_{0}$ is a vector describing a point on the interface. After a time $\Delta t$, the A particles have moved on average a distance $\vec{F}^{(A)} \Delta t / \xi$, while the B particles have been displaced by a distance $\vec{F}^{(B)} \Delta t / \xi$. Neglecting any collisions, the A particles near the interface will move towards a new interface, which is described by the set of vectors

$$
\vec{r}^{(A)}(s)=\vec{R}_{0}+s \vec{d}+\vec{F}^{(A)} \Delta t / \xi,
$$

while the B particles near the interface will drift to

$$
\vec{r}^{(B)}\left(s^{\prime}\right)=\vec{R}_{0}+s^{\prime} \vec{d}+\vec{F}^{(B)} \Delta t / \xi
$$

with another real parameter $s^{\prime}$. The interface can only be stable if these two interfaces coincide. If they do not, there is either empty space, which will be filled by neighbouring particles, or A and B particles will collide, which will destroy the interface as well. Hence, the stability criterion is that for any $s$ there is an $s^{\prime}$ such that $\vec{r}^{(A)}(s)=\vec{r}^{(B)}\left(s^{\prime}\right)$, which simply yields the condition

$$
\vec{d}=\frac{\Delta t}{\xi\left(s-s^{\prime}\right)}\left[\vec{F}^{(B)}-\vec{F}^{(A)}\right] .
$$

This implies that for an interface to be stable, its direction has to be collinear with the force difference

$$
\Delta \vec{F}=\vec{F}^{(B)}-\vec{F}^{(A)} .
$$

Hence, the angle $\alpha$ describing the interface orientation (see figure 1) is

$$
\alpha=\arcsin \frac{\Delta \vec{F} \cdot \vec{F}^{(B)}}{|\Delta \vec{F}|\left|\vec{F}^{(B)}\right|} .
$$

Clearly, in contrast to the case of parallel forces, the interface position will move in space. The interface velocity $v_{I}$ normal to its position can be calculated as

$$
v_{I}=\frac{\left|\vec{F}^{(A)} \times \vec{F}^{(B)}\right|}{\xi|\Delta \vec{F}|} .
$$


Obviously, the same argument can be repeated with exchanged roles of A and B, showing that stable parallel lanes with the direction $\Delta \vec{F}$ are expected to move, with the interface velocity $v_{I}$ given by equation (10). Furthermore, the same argument applied for a small $\Delta t$ shows that a curved interface is unstable, such that a stable interface has to be straight.

Let us finally discuss two special cases. First returning to parallel forces, indeed the interface direction is parallel to the field direction, the angle $\alpha$ is $\pi / 2$ and the interface velocity vanishes as follows directly from equation (10). Second the case of perpendicular forces deserves some particular attention. Here the angle $\alpha$ is

$$
\alpha=\arctan \frac{\left|\vec{F}^{(B)}\right|}{\left|\vec{F}^{(A)}\right|}
$$

and the interface velocity can be expressed as

$$
v_{I}=\frac{\left|\vec{F}^{(A)}\right|\left|\vec{F}^{(B)}\right|}{\xi|\Delta \vec{F}|} .
$$

Obviously, this general argument is only a necessary condition for a stable AB interface. An alternative is a mixed situation with no interface at all driven by entropy. In analogy to the parallel case we anticipate that a critical strength of $\Delta \vec{F}$ is needed to build stable lanes. This will now be investigated by computer simulation and simple theory in more detail.

\subsection{Computer simulation results}

In our simulation we assume-without loss of generality-the direction of $\vec{F}^{(B)}$ along the $y$ axis of the simulation box. The model parameters are fixed to $\rho \sigma^{2}=1.0, \kappa=4.0$ and $V_{0}=2.5 k_{B} T$. Simulation snapshots for different external field strengths are shown in figures 2(a)-(d).

In figure 2(a), no field is applied and a homogeneous completely mixed state is visible. In figure $2(\mathrm{~b})$, on the other hand, the external forces are parallel: $\vec{F}^{(A)}=-\vec{F}^{(B)}$. The magnitude $\left|\vec{F}^{(A)}-\vec{F}^{(B)}\right|$ is beyond the critical strength [17] such that lane formation parallel to the field shows up. Nonparallel forces with perpendicular directions are investigated in figures 2(c) and (d). One observes formation of tilted lanes, which are indeed in the direction of the force difference vector as expected from our general argument. In following the configurations as a function of time we verified the simple formula of the interface velocity $v_{I}$ as given in equation (10).

At this point we want to comment on the layer thickness. From the simulations, depending on the initial conditions, a broad distribution of different thicknesses is obtained. The final stable steady state is expected to be a system completely separated into two large phaseseparated regions such that a finite thickness of the lanes is only transient. Of course, this has to be checked by further more time-consuming simulations.

In a next step, we study perpendicular forces maintaining their ratio $q=\left|\vec{F}^{(A)}\right| /\left|\vec{F}^{(B)}\right|$ fixed but increasing their magnitude. For small forces the system remains demixed. In increasing the strength of both fields, we have calculated suitable order parameters which are sensitive to tilted lane formation. These are immediate generalizations of those used in [17]. The order parameter exhibits a sharp jump, indicating the critical field strength of the force difference vector upon which tilted lane formation is achieved. There is a clear hysteresis loop if the force is reduced again such that the nonequilibrium phase transition towards tilted lane formation is of first order. The critical value $\Delta F_{c}$ of the force difference $|\Delta \vec{F}|$ is shown versus the ratio $q=\left|\vec{F}^{(A)}\right| /\left|\vec{F}^{(B)}\right|$ in figure 3 . We shall compare these data with a simple theoretical prediction in the next subsection. 
(a)

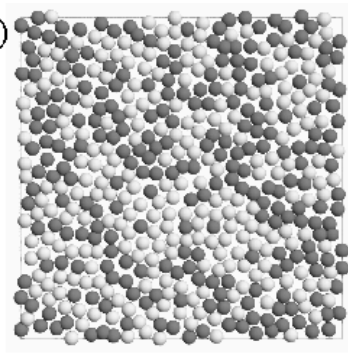

(c)

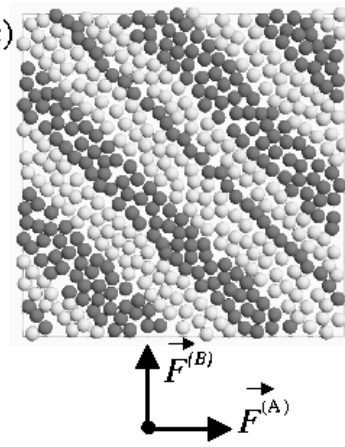

(b)

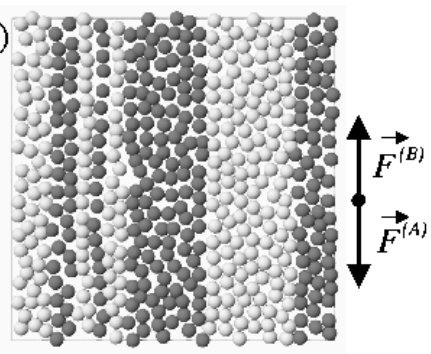

(d)

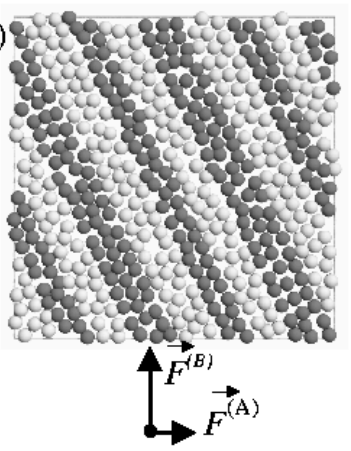

Figure 2. Typical simulation snapshots of the two-dimensional system: (a) disordered state without field; (b) lane formation with parallel fields $\vec{F}^{(A)}=-\vec{F}^{(B)}=180 k_{B} T / \sigma$ in the $y$-direction above the critical force; (c) lane formation with perpendicular fields of the same magnitude $\left|\vec{F}^{(A)}\right|=\left|\vec{F}^{(B)}\right|=180 k_{B} T / \sigma$ above the critical force difference; (d) lane formation with perpendicular fields and $\left|\vec{F}^{(A)}\right| /\left|\vec{F}^{(B)}\right|=1 / 2$ with $\left|\vec{F}^{(B)}\right|=180 k_{B} T / \sigma$ above the critical force difference. In (c) and (d) the A particles are drifting in the $x$-direction, while B particles are drifting in the $y$-direction. The lanes are moving perpendicular to $\Delta \vec{F}$. The particles are depicted as spheres with diameter $\sigma$. A light sphere is an A particle while a grey sphere is a B particle.

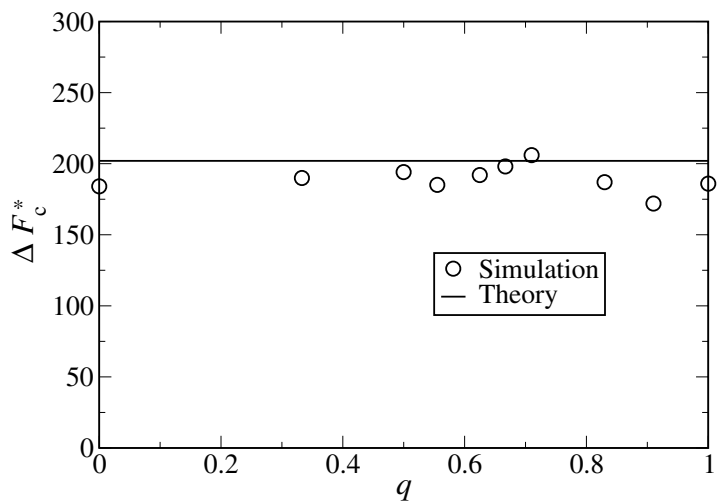

Figure 3. Dimensionless critical force difference $\Delta F_{\mathrm{c}}^{*}=\Delta F_{\mathrm{c}} \sigma / k_{B} T$ versus the ratio $q$ between the perpendicular forces $\left|\vec{F}^{(A)}\right|$ and $\left|\vec{F}^{(B)}\right|$ for the two-dimensional system. Above the critical force the system is in the patterned state characterized by stripe formation. The circles are simulation results, while the solid line is theory. The parameters are $\kappa=4.0, V_{0}=2.5 k_{B} T$ and $\rho \sigma^{2}=1.0$.

A careful remark is in order for small positive $q$. The periodic boundary conditions used in the simulation correspond to a toroidal topology shown in figure 4 . If $q$ becomes small the 


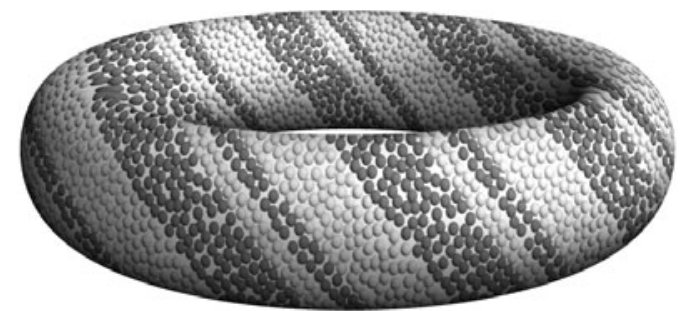

Figure 4. Visualization of the two-dimensional system by mapping a two-dimensional simulation snapshot onto the surface of a torus. A particles are drifting along the torus, while B particles are drifting around it. The lanes are moving perpendicular to their direction.

boundary conditions enforce a multiple winding around the torus such that finite-size effects are expected to be significant. Therefore, we have not shown simulation data for small $q$ in figure 2 .

\subsection{Theory}

We are aiming at a rough theoretical estimation of the boundaries of the demixing transition with constant external fields $\vec{F}^{(A)}$ and $\vec{F}^{(B)}$. This was put forward in [17] for parallel forces and is generalized here to the general case of nonparallel forces. Consider first a central collision between an A and B particle pair (see figure 5). Transforming the trajectories onto one with a fixed common centre of mass of the two particles, one realizes that the collision is effectively driven by half of the force difference, $\frac{1}{2}\left(\vec{F}^{(A)}-\vec{F}^{(B)}\right)=-\frac{1}{2} \Delta \vec{F}$ for A particles and $\frac{1}{2}\left(\vec{F}^{(B)}-\vec{F}^{(A)}\right)=\frac{1}{2} \Delta \vec{F}$ for B particles. A transition towards patterned lanes is expected if $|\Delta \vec{F}| / 2$ is larger than a typical average force between $\mathrm{A}$ and $\mathrm{B}$ particles, and lane formation is induced. The latter force depends both on density and on the external fields themselves. We estimate a typical average force between two opposite particles by considering different 'effective' interparticle spacings. The first typical interparticle spacing is set by the density alone, $a=\rho^{-1 / 2}$. Including fluctuations in the interparticle distance induced by a finite temperature results in a further smaller effective average distance $\tilde{a}$ as obtained by setting a typical interparticle energy equal to $V(a)+k_{B} T$. Hence $\tilde{a}=V^{-1}\left[V(a)+k_{B} T\right]$ where $V^{-1}$ is the inverse function of the interaction potential $V(r)$. Finally the presence of the external fields enforces an even smaller averaged distance $a^{\prime}$ between two colliding opposite particles.

We estimate this minimum distance $a^{\prime}$ by adding the net force per colliding particle $\Delta F / 2$ to the force at distance $\tilde{a}$ via

$$
a^{\prime}=F^{-1}[\Delta F / 2+F(\tilde{a})],
$$

where $F^{-1}$ is the inverse function of $F(r)=-\frac{\mathrm{d}}{\mathrm{d} r} V(r)$. In general, an AB particle pair will not collide directly along $\Delta \vec{F} / 2$ such that the actual average distance is between $a^{\prime}$ and $\tilde{a}$. Hence the averaged force $\bar{f}$ between an $\mathrm{A}$ and a B particle is roughly

$$
\bar{f}=\frac{1}{\tilde{a}-a^{\prime}}\left[V\left(a^{\prime}\right)-V(\tilde{a})\right] .
$$

The critical force difference $\Delta F_{\mathrm{c}}$ is reached when it becomes of the order of the mean force $\bar{f}$,

$$
\Delta F_{\mathrm{c}}=2 \lambda \bar{f}
$$

$\lambda=2$ is a dimensionless prefactor, which is determined by an optimal fit to all simulation results for parallel forces [17]. 


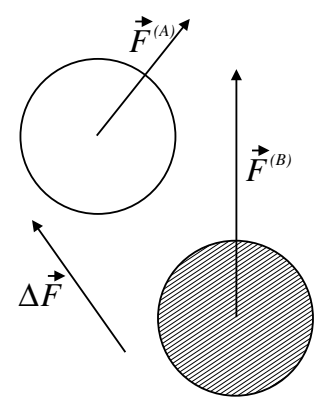

Figure 5. Sketch of two colloids of opposite type colliding due to the external fields $\vec{F}^{(A)}$ and $\vec{F}^{(B)}$. The A particle is white, while the B particle is grey. The particles collide effectively in the direction of the difference force $\Delta \vec{F}=\vec{F}^{(B)}-\vec{F}^{(A)}$ as seen from a fixed centre of mass.

The basic prediction of this simple theory is that the only essential parameter governing tilted lane formation is the magnitude $|\Delta \vec{F}|$. This prediction can be tested by simulation. In fact, in figure 3, the critical value of $|\Delta \vec{F}|$ is shown for different ratios $q=\left|\vec{F}^{(A)}\right| /\left|\vec{F}^{(B)}\right|$. Were the theory correct, all the simulation data should fall on a horizontal line independent of $q$. As can be deduced from figure 3, this is indeed confirmed. Furthermore, the actual magnitude predicted from the theory is in line with the simulation data. Note that -as far as the nonparallel case is concerned-there is no fit parameter involved. The global fit parameter $\lambda$ is solely adjusted to the case of parallel forces.

\subsection{Three-dimensional model}

The model and all methods and arguments can readily be generalized to three spatial dimensions. Similar conclusions hold for the formation of tilted lanes. We have also performed computer simulations in a cubic box in three dimensions and observed tilted lane formation. Results are presented in figure 6: tilted lane formation is clearly visible in the plane spanned by the two forces $\vec{F}^{(A)}$ and $\vec{F}^{(B)}$ (see figure 6(a)). Perpendicular to the direction $\Delta \vec{F}$ of the lanes, the system shows a structure reminiscent of two-dimensional spinodal decomposition (see figure 6(b)). The parameters are for these snapshots $\kappa=4.0, V_{0}=2.5 k_{B} T$ and $\rho \sigma^{3}=1$. In conclusion, this shows that pattern formation is a general effect, which is independent of the dimensionality of the model.

\section{Re-entrant freezing for a driven Brownian crystal}

In this section we focus on a Brownian crystal which is driven by an external field. To this end, the external fields acting on A and B particles are parallel $\left(\vec{F}^{(A)}=-\vec{F}^{(B)}=f \vec{e}_{y}\right)$ but in contrast to [17] the field-free equilibrium initial state is a triangular crystal. The case of a fluid field-free configuration is easier as rotational symmetry with respect to the direction of the external field applied is ensured. This is no longer true for a crystal where one has to specify the field direction with respect to the crystalline orientation, resulting in an anisotropy which reflects the crystalline symmetry. We performed simulations of the two-dimensional system where we have chosen a fixed relative orientation of the external field with respect to the crystal lattice. The actual value of the critical field should depend on this orientation. Clearly, in the absence of any external field, A and B particles are indistinguishable; hence, the equilibrium state is a randomly occupied triangular crystal. In the other limit of very strong fields, one expects again phase separation into completely demixed A and B regions. Once they 
(a)

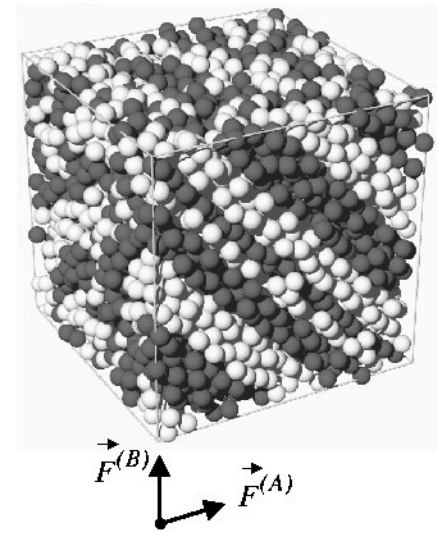

(b)

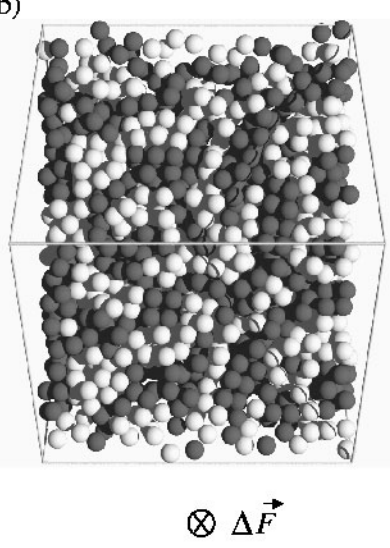

Figure 6. Typical snapshots of the three-dimensional system with perpendicular external fields above the critical force difference. The magnitude of the forces is $\left|\vec{F}^{(A)}\right|=\left|\vec{F}^{(B)}\right|=150 k_{B} T / \sigma$. (a) Three-dimensional view, (b) view of the plane perpendicular to the $\Delta \vec{F}$ vector.

are demixed, they follow Boltzmann statistics. Consequently the equilibrium state is a pure A (or B) crystal of the same lattice as the original (field-free) one. What is less clear intuitively is how the system transforms from the first randomly occupied crystal into the demixed crystal if the field is turned continuously on. At least two scenarios are conceivable: either the system retains the underlying solid lattice but particle exchange hopping processes generated by the external field demix the crystalline state, or the crystal first melts mechanically via the external field and then crystallizes again. In our two-dimensional simulations we almost exclusively observed the latter scenario.

In order to detect a triangular crystalline order in two dimensions we define a suitable crystallinity order parameter $\Psi_{6}$ that probes sixfold symmetry around a given particle via $[32,33]$

$$
\Psi_{6}=\mid\left\langle\frac{1}{12 N} \sum_{j=1}^{2 N} \sum_{\langle k\rangle} \mathrm{e}^{6 \mathrm{i} \phi_{j k}}\right\rangle .
$$

Here the $k$-sum includes the six nearest neighbours of the given particle and the $j$-sum extends over $2 N$ particles in the simulation box. The large angular brackets indicate a time average. $\phi_{j k}$ is the polar angle of the interparticle distance vector with respect to a fixed reference frame. For ideal sixfold symmetry, i.e. for a perfect triangular crystal, $\Psi_{6}=1$. Thermal fluctuations cause deviations from this ideal case but a value of $\Psi_{6}>0.8[32,33]$ is conveniently taken to be conclusive evidence for a triangular crystal.

In figure 7 we plot $\Psi_{6}$ versus the external force difference $f^{*}=\left|\vec{F}^{(B)}\right| \sigma / k_{B} T$ for fixed parameters $\kappa=4.0, V_{0}=15.0 k_{B} T$ and $\rho \sigma^{2}=1$. The direction of the force is the (11)direction of the triangular crystal. Note that, in contrast to the parameters used in section 3 , the interaction energy is much larger, to ensure that the equilibrium field-free state is crystalline. Indeed the crystallinity order parameter in the field-free case is around $\Psi_{6}=0.87$. Upon increasing the external field strength to $f^{*} \approx 10$, the crystallinity order parameter drops sharply and stays close to zero, indicating a melting of the crystal induced by the external field. This melting process is mainly caused by a mechanical stress induced by the external field with respect to $\mathrm{AB}$ particle pairs. As the field becomes larger $\left(f^{*} \gtrsim 200\right)$, the order parameter $\Psi_{6}$ increases again to values close to 0.7 . This is accompanied by lane formation as indicated 


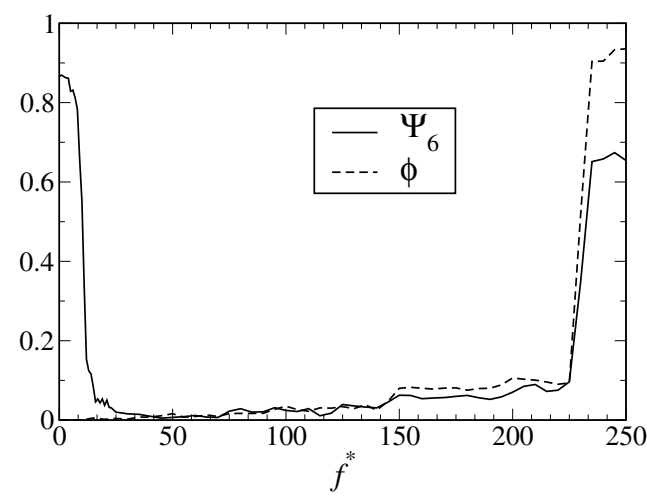

Figure 7. Crystallinity order parameter $\Psi_{6}$ (solid curve) and lane order parameter $\phi$ (dashed curve) plotted versus reduced external force $f^{*}$ as calculated by a Brownian dynamics simulation.

by a drastic increase of another order parameter $\phi$ defined in [17] which is sensitive to lane formation. The whole scenario is also illustrated by simulation snapshots shown in figure 8 , from which the direction of the external force with respect to the crystal orientation can be read off. While figure 8(a) corresponds to a field-free randomly mixed crystal, figures 8(b) and (c) are in the molten state while figure 8(d) represents refrozen demixed crystals sliding against each other. In figures 8(b) and (c) wormlike structures along the fields occur as a precursor to lanes formed by solids. Consequently we have shown evidence for a re-entrant freezing behaviour generated by external fields in nonequilibrium. Obviously all the previous results should also be observable in three dimensions. A qualitatively similar situation occurs for colloidal solids in linear shear flow [34-36]. A continuous increase of the shear rate can lead to shear melting and subsequent recrystallization into a different solid structure [37-39]. A similar effect is shear thinning and subsequent shear thickening as observed in colloidal fluids for increasing shear rates [40].

\section{Conclusions}

To summarize: we have generalized earlier studies of nonequilibrium pattern formation in continuum-driven diffusive mixtures to nonparallel external fields and crystalline states. As main results we found tilted lanes along the force difference vector, which are wandering with a constant interface velocity provided the external force difference is large enough. Furthermore, a solid melts and refreezes if the magnitude of an external field is increased.

Let us remark on possibilities of nonequilibrium lane formation in more complicated systems. First, the system we studied was completely symmetric, involving the same partial densities and the same particle-particle interactions. For experimental realizations [19-22] this will not be fulfilled in general. However, the basic physics of lane formation will not change. Second, if ternary and further multicomponent mixtures beyond binary ones are considered, we expect cascades of lane formation transitions involving the different particle species as the external field is increased.

We finally comment on possible experimental realizations of our model: there are different fields where the pattern formation we predicted within our model can be verified, namely in colloidal dynamics and in pedestrian motion. Binary colloidal mixtures indeed can be driven by constant external forces. Important examples for parallel forces are sedimentation, where the external force is gravity [19,20], or electrokinetic motion of charged colloids [41], where the 
(a)

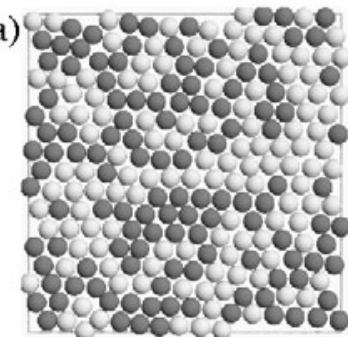

(b)

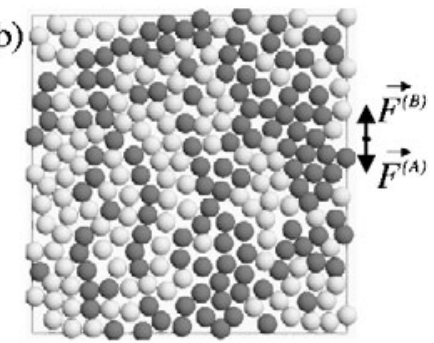

(d)

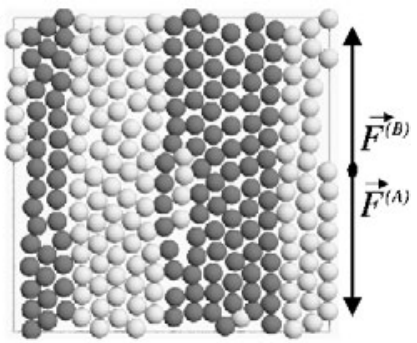

Figure 8. Snapshots of the two-dimensional system for different external forces, starting with a solid in the free-field state. The forces acting on the two different particle types are pointing into opposite directions. The forces are (a) $f^{*}=0$, (b) $f^{*}=50$, (c) $f^{*}=150$ and (d) $f^{*}=250$. In (a) and (d) the system shows a solid structure, while in (b) and (c) the system is a homogeneous fluid. Here the total number of particles is $2 N=250$.

external force is an electric field. Both the fluid and crystalline field-free state can be studied. A recent realization with mixed crystals can be found in [42]. One possible drawback is the hydrodynamic backflow [43] caused by strong hydrodynamic interactions [44-46], which were neglected in our model. An overall backflow can be avoided by a time-dependent oscillatory field (e.g. AC electric field), which leads qualitatively to the same lane formation if its frequency is small enough [17]. We further think that the long-ranged hydrodynamic flow around a driven colloidal particle will favour lane formation, i.e. the critical field strength needed to generate lane formation is expected to be lower than with hydrodynamic interactions neglected. Colloids can also be exposed to external laser-optical and magnetic fields [10], which generate external forces in a controlled way coupling to the dielectricity (respectively the magnetic permeability) of the colloidal material. Nonparallel external forces in colloidal mixtures can be realized by crossing two external fields e.g. gravity with electric, laser-optical with electric, laser-optical with magnetic etc. The two species of a colloidal mixture will in general respond differently to the two external fields such that the resulting total external force will be different in direction.

A different realization is dynamics of pedestrians in pedestrian zones and in lecture halls. Similar off-lattice models involving Brownian particles have been used to simulate the collective behaviour of pedestrians [12,13], including escape panic [47]. Our setup of perpendicular external fields is realized by two crossing pedestrian lanes in which pedestrians are only moving in one direction. Based on our results, we would expect tilted lane formation provided the density of the pedestrians is high enough. Finally it would be interesting to extend phenomenological hydrodynamical theories which predict lane formation for parallel forces via an instability $[20,48-53]$ to the case of tilted forces. Work along these lines is in progress. 


\section{Acknowledgments}

It is with great pleasure that we dedicate this paper to J P Hansen on the occasion of his 60th birthday. We thank T Palberg, J Chakrabarti, J Sherwood, V Popkov and E Allahyarov for helpful remarks. Financial support from the DFG (Sonderforschungsbereich 237) is gratefully acknowledged.

\section{References}

[1] Cross M C and Hohenberg P C 1993 Rev. Mod. Phys. 65851

[2] van Saarloos W 1998 Phys. Rep. 3019

[3] Allen M P and Tildesley D J 1989 Computer Simulations of Liquids (Oxford: Clarendon)

[4] Baus M, Rull L and Ryckaert J (eds) 1995 Observations, Prediction and Simulation of Phase Transitions in Complex Fluids (Series B: Physics) (Dordrecht: Kluwer)

[5] Löwen H 1994 Phys. Rep. 237249

[6] Hansen J P and McDonald I R 1986 Theory of Simple Liquids 2nd edn (London: Academic)

[7] Schmittmann B and Zia R K P 1995 Phase Transitions and Critical Phenomena vol 17, ed C Domb and J Lebowitz (London: Academic)

[8] Ramaswamy S 1999 Curr. Sci. 77402

[9] Trappe V, Prasad V, Cipelletti L, Segre P N and Weitz D A 2001 Nature 411772

[10] Löwen H 2001 J. Phys.: Condens. Matter 13 R 415

[11] Murray C A and Grier D G 1996 Annu. Rev. Phys. Chem. 47421

[12] Helbing D, Farkas I J and Vicsek T 2000 Phys. Rev. Lett. 841240

[13] Helbing D, Molnár P, Farkas I J and Bolay K 2001 Environ. Planning B 28361

[14] Burstedde C, Klauck K, Schadschneider A and Zittartz J 2001 Physica A 295507

[15] Hoffmann G P and Löwen H 1999 Phys. Rev. E 603009

[16] Hoffmann G P and Löwen H 2001 J. Phys.: Condens. Matter 139197

[17] Dzubiella J, Hoffmann G P and Löwen H 2002 Phys. Rev. E 65021402

[18] Rothman D H and Zaleski S 1994 Rev. Mod. Phys. 661417

[19] Weiland R H, Fessas Y P and Ramaro B V 1984 J. Fluid. Mech. 142383

[20] Batchelor G K and van Rensburg R W J 1986 J. Fluid. Mech. 166379

[21] Nasr-El-Din H A, Masliyah J H and Nandakumar K 1999 Can. J. Chem. Eng. 111003

[22] Yan Y and Masliyah J H 1993 Int. J. Multiphase Flow 19875

[23] Löwen H and Kramposthuber G 1993 Europhys. Lett. 23673

[24] Chang E and Hone D W 1988 Europhys. Lett. 5635

[25] Löwen H 1992 J. Phys.: Condens. Matter 410105

[26] Löhle B and Klein R 1997 Physica A 235224

[27] Naidoo K J and Schnitker J 1994 J. Chem. Phys. 1003114

[28] Hoffmann G P and Löwen H 2000 J. Phys.: Condens. Matter 127359

[29] Löwen H, Hansen J P and Roux J N 1991 Phys. Rev. A 441169

[30] Ermak D L 1975 J. Chem. Phys. 624189

[31] Santra S B, Schwarzer S and Herrmann H 1996 Phys. Rev. E 545066

[32] Heni M and Löwen H 2000 Phys. Rev. Lett. 853668

[33] Heni M and Löwen H 2001 J. Phys.: Condens. Matter 134675

[34] Imhof A, van Blaaderen A and Dhont J K G 1994 Langmuir 103477

[35] Palberg T and Würth M 1996 J. Physique 6237

[36] Onuki A 1997 J. Phys.: Condens. Matter 96119

[37] Stevens M J and Robbins M O 1993 J. Chem. Phys. 982319

[38] Lahiri R and Ramaswamy S 1994 Phys. Rev. Lett. 731043

[39] Lahiri R and Ramaswamy S 1996 Physica A 22484

[40] Newstein M C, Wang H, Balsara N P, Lefebvre A A, Shnidman Y, Watanabe H, Osaki K, ShikitaT, Niwa H and Morishima Y 1999 J. Chem. Phys. 1114827

[41] Mantegazza F, Degiorgio V, Delgado A V and Arroyo F J 1998 J. Chem. Phys. 1096905

[42] Wette P, Schope H J, Biehl R and Palberg T 2001 J. Chem. Phys. 1147556

[43] Segre P N, Liu F, Umbanhowar P and Weitz D A 2001 Nature 409594

[44] Nägele G 1996 Phys. Rep. 272215

[45] Dhont J K G 1996 An Introduction to Dynamics of Colloids (Amsterdam: Elsevier) 
[46] Ladd A J C 1993 Phys. Fluids A 5299

[47] Helbing D, Farkas I J and Vicsek T 2000 Nature 407487

[48] Kynch G J 1952 Trans. Faraday Soc. 48166

[49] Valiveti P and Koch D L 1998 Appl. Sci. Res. 58275

[50] Valiveti P and Koch D L 1999 Phys. Fluids 113283

[51] Burger R, Concha F, Fjelde K K and Karlsen K H 2000 Powder Technol. 11330

[52] Biesheuvel P M, Verweij H and Breedveld V 2001 AIChE J. 4745

[53] Chakrabarti J, Dzubiella J and Löwen H 2002 to be published 